J. Austral. Math. Soc. 19 (Series B), (1975), 66-80.

\title{
ON AIR FLOW OVER FREE SURFACES OF STATIONARY WATER
}

\author{
E. O. TUCK
}

(Received 12 November 1974)

(Revised 5 March, 1975)

\begin{abstract}
A correspondence is established between flows of air above stationary water, and flows of water below air at atmospheric pressure. Flows in the latter category are well studied, and all such hydrodynamic flows can be "turned upside-down" to generate flows of air in which the free surface deforms under gravity, due to a balance between aerodynamic and hydrostatic pressures. Examples are given of some exact inverse solutions, and a general semiinverse approach is outlined for numerical solutions via an integral formulation.
\end{abstract}

\section{Introduction}

In almost all of classical water-wave theory (e.g. Wehausen and Laitone, [13]) it is implicitly or explicitly assumed that movement of the air above the free surface has no effect on the hydrodynamics, so that the free surface may be treated as a surface of constant (atmospheric) pressure. If one wished to be more accurate, one could consider a coupled dynamic problem in which both air motion and water motion were taken into account, the condition at the free surface (for inviscid fluids) being now continuity of pressure.

It is then clear that significant coupling between air and water motions occurs if and only if the dynamic contributions $-\frac{1}{2} \rho q^{2}$ to the pressure are comparable in the two fluids, where $\rho$ is density and $q$ fluid velocity magnitude. Thus the condition for validity of the classical water-wave theory, neglecting air motion, is that the velocity of the air is significantly less than $\Delta^{-\frac{1}{2}}$ times the velocity of the water, where $\Delta$ is the air/water density ratio. Since $\Delta \simeq 0.0012$, the classical theory remains valid up to air velocities several times as great as the water velocity.

In the present paper we are concerned with other extreme, when the air velocity is so far in excess of the water velocity, that $\frac{1}{2} \rho q^{2}$ in the air far exceeds its value in the water. This requires for example that the water velocity be 
much less than $4 \%$ of the air velocity. If this condition is met, the motion on the water may be neglected when computing the motion of the air. The condition at the free surface is however not one of constant pressure, but one of hydrostatic pressure.

Problems of this nature occur in a number of widely-varying applications. Perhaps the most obvious potential application is to oceanographic problems such as sea-level variations due to meteorological conditions. Such phenomena as the "inverse barometer" effect are well known (e.g. Hamon, [4]). On a somewhat smaller geophysical scale, problems associated with sea-level variations due to land or sea breezes at a shore line are typical of the type of problem of greatest interest here, in which interactions between free and solid surfaces are dominant. Unfortunately, however, in such geophysical applications it is seldom permissible to neglect viscosity, as we shall do in the following analysis.

In an engineering context, problems of impingement of jets of fluid on a free surface have been studied theoretically and experimentally by a number of authors (e.g. Berghmans, [1], Turkdogan [11], Olmstead and Raynor [8]). Some of this interest results from potential applications to welding, and other problems in metallurgy.

Finally, (but not exclusively) there are applications in naval hydrodynamic contexts, particularly to the dynamics of hovercraft or similar vehicles. Hydrodynamicists generally approximate hovercraft by given pressure distributions travelling over a classical free surface. But the pressure distribution of a hovercraft, at least in the edge or seal region, must depend on the displacement of the water beneath it, and therefore it is dangerous to treat it as given. Indeed, some authors (e.g. Doctors [2]) have found it necessary to "smooth off" pressure distributions in an artificial and empirical manner near the edges, in order to obtain finite wave resistances. Murthy [5] considered more fundamental questions involving the static depression of the water surface beneath a given pressure distribution, allowing for surface tension, and also made some empirical estimates of the actual dynamic effect of the air flow on the water surface. The present analysis is relevant only to hovercraft without forward motion, since the water is assumed stationary.

In the following sections we formulate and discuss some very limited and idealized problems of air flow over free surfaces of stationary water. Only two-dimensional steady flows are considered, and no account is taken of viscosity or compressibility of the air. Surface tension is included at first, but neglected subsequently. The resulting problems are still in every respect as complicated as those of classical water-wave theory.

In fact, the resulting problems are in a certain sense complementary to water-wave problems, since the resulting free-surface condition is obtained 
from the water-wave condition by replacing the acceleration of gravity $g$ with $-g / \Delta$. Thus the aerodynamic problems are negative-gravity water-wave problems turned upside-down! Once this observation is made, the whole literature on water-wave problems can be extended to cover aerodynamic free-surface problems.

Hōwever, most problcms of present interest represent violent jet-like air flows, or possess sharp-cornered body geometries, so that the free-surface condition remains essentially non-linear, in contrast to the water-wave case, where linearization has been particularly fruitful. Little assistance can therefore be gained by analogy, although part of the present paper involves exploiting inverse solutions analogous to those well-established in non-linear hydrodynamic free-surface problems.

A common feature of two-dimensional aerodynamic and hydrodynamic free-surface problems is that they can be reduced to non-linear integral equations. The classical examples of the use of such a reduction in water-wave theory are the proofs of existence of periodic water-waves. Unfortunately, little use has been made of these integral equations, even in the hydrodynamic case, as tools for obtaining numerical solutions of potentially-practical problems involving interactions between free and solid surfaces, although Olmstead and Raynor [8] have made progress in such an attack on a jet impingement problem. Work is proceeding on numerical solution of integral equations of the kind presented here.

\section{The non-linear free-surface conditions}

We consider two-dimensional steady flow of an incompressible fluid (e.g. air at sufficiently low Mach number) of density $\rho_{A}$ over a stationary incompressible fluid (e.g. water) of density $\rho_{w}$. The interface between the two fluids is a free surface, with equation $y=\eta(x)$. The kinematic boundary condition is that this surface be a stream surface, i.e.

$$
\phi_{y}=\eta^{\prime} \phi_{x}
$$

where $q=\nabla \phi$ is the fluid velocity vector, or

$$
\psi=0
$$

where $\psi$ is the stream function, conjugate to $\phi$. Note that flow takes place in the air only, so that $\phi, \psi$ are harmonic for $y \geqq \eta(x)$; thus $f(z)=\phi+i \psi$ is an analytic function of $z=x+i y$, for $y \geqq \eta(x)$.

The dynamic free-surface condition is that any jump in pressure across $y=\eta$ is supported only by surface tension. Now in the water, which is stationary, the pressure is hydrostatic, i.e.

$$
p_{w}=p_{0}-\rho_{w} g y
$$


where $p_{0}$ is some base pressure, equal to atmospheric pressure if $y=0$ at some portion of the free surface where the air is at rest. In the moving air, the pressure is given by Bernoulli's equation as

$$
p_{A}=p_{0}-\frac{1}{2} \rho_{A}|q|^{2}-\rho_{A} g y,
$$

where $p_{0}$ is the same base pressure as in (2.4), since again again $p_{A}=p_{0}$ where $y=0$ and $q=0$.

Now the jump in pressure across $y=\eta$ must be given by

$$
p_{w}-p_{A}=-T \eta^{\prime \prime}(x) /\left[1+\left(\eta^{\prime}\right)^{2}\right]^{\frac{1}{2}}
$$

where $T$ is the surface tension, i.e.

$$
-\left(\rho_{w}-\rho_{A}\right) g \eta+\frac{1}{2} \rho_{A}|q|^{2}=-T \eta^{\prime \prime} /\left[1+\left(\eta^{\prime}\right)^{2}\right]^{\frac{3}{2}}
$$

or, since $\rho_{A} \ll \rho_{W}$,

$$
g \eta-\frac{1}{2} \Delta|q|^{2}-\frac{T}{\rho_{w}} \frac{\eta^{\prime \prime}}{\left(1+\left(\eta^{\prime}\right)^{2}\right]^{\frac{2}{2}}}=0
$$

where

$$
\Delta=\rho_{A} /\left(\rho_{w}-\rho_{A}\right) \simeq \rho_{A} / \rho_{w}
$$

is the density ratio. Equation (2.6) constitutes the required free-surface condition for static water. This condition may also be interpreted as an approximation to the condition which holds when both fluids are moving, providing $\rho_{A}\left|\boldsymbol{q}_{A}\right|^{2} \gg \rho_{W}\left|\boldsymbol{q}_{W}^{2}\right|$, where $\boldsymbol{q}_{A}=\boldsymbol{q}$ is the air velocity, and $\boldsymbol{q}_{W}$ the water velocity.

Note that the zero level,$\eta=0$ of the free surface has been normalized to that for zero air flow. The corresponding uniform level for a uniform flow $|\boldsymbol{q}|=U$ is

$$
\eta_{0}=\frac{U^{2} \Delta}{2 g}
$$

The length scale $\eta_{0}$ is the amount by which the free surface is raised due to the lower free-stream pressure when the air is blowing. It is important to note that, if $U$ is a typical measure of $q$, we shall take $\eta_{0}$ as our typical length scale. This means that, even though the density ratio $\Delta$ is very small, typically 0.0012 for air-water interfaces, nevertheless on a length scale of $\eta_{0}$ the first two terms of (2.6) are comparable in magnitude. Indeed, if we perform the nondimensionalization $x=\eta_{0} x^{*}, \eta=\eta_{0} \eta^{*}, q=U q^{*}$ etc., we have from (2.6)

$$
\eta^{*}\left|q^{*}\right|^{2}-T^{*} \frac{d^{2} \eta^{*}}{d x^{* 2}}\left[1+\left(\frac{d \eta^{*}}{d x^{*}}\right)^{2}\right]^{-\frac{3}{2}}=0
$$


where

$$
T^{*}=\frac{T}{\left(\rho_{W}-\rho_{A}\right) g \eta_{0}^{2}} \simeq \frac{4 T g \rho_{w}}{U^{4} \rho_{A}^{2}}
$$

is a non-dimensional surface tension.

It is of interest to contrast the corresponding boundary condition for conventional non-linear water waves, in which the water $y<\eta(x)$ moves, but the effect of the air above it is negiigibie. Then (Wehausen and Laitone, [13], p. 453) the free-surface condition corresponding to (2.6) would be simply

$$
g \eta+\frac{1}{2}|q|^{2}-\frac{T}{\rho_{w}} \eta^{\prime \prime}\left[1+\left(\eta^{\prime}\right)^{2}\right]^{-\frac{1}{2}}=0
$$

and the normalized equation corresponding to (2.9) would be

$$
\eta^{*}+\left|\boldsymbol{q}^{*}\right|^{2}-T^{*} \frac{d^{2} \eta^{*}}{d x^{* 2}}\left[1+\left(\frac{d \eta^{*}}{d x^{*}}\right)^{2}\right]-\frac{3}{2}=0
$$

Clearly all that has happened to the dimensionless equation is a switch in the sign of the velocity term. In the absence of surface tension, the dimensional aerod ynamic condition (2.b) is obtained from the hydrodynamic condition (2.11) by replacing $g$ with $-g / \Delta$. The apparent dimensional gravity is of course much increased, because of the factor $\Delta^{-1}$, but on the length scale $\eta_{0}$ this increase scales out, as is clear by comparing the dimensionless equations (2.9), (2.12). A reflection of this property is that, even including surface tension, the hydrodynamic condition (2.11) can be retrieved from the aerodynamic condition (2.6) by the artificial choice $\Delta=-1$. We shall exploit this property in the following sections.

In fact, every hydrodynamic $(\Delta=-1)$ flow can be turned upside-down, reversed in direction, and increased in magnitude, to generate an aerodynamic $(\Delta>0)$ counterpart. Thus if we use a subscript "-" to denote quantities satisfying (2.11), then

$$
q(x, y)=\Delta^{-\frac{1}{2}} q_{-}(-x,-y)
$$

satisfies (2.6) on $y=\eta(x)$, where

$$
\eta(x)=-\eta_{-}(-x),
$$

and defines a flow of air in $y \geqq \eta(x)$. This correspondence allows us to make use of the large range of hydrodynamic solutions to generate new air-blowing solutions.

\section{The linearized problem}

Suppose we set

$$
\phi=U x+\phi_{1}
$$


and

$$
\eta=\eta_{0}+\eta_{1}
$$

where $\left|\nabla \phi_{1}\right| \ll U$ and $\left|\eta_{1}\right| \ll \eta_{0}$. That is, we consider only small perturbations to a uniform air flow $U$ over a flat free surface at $y=\eta_{0}$. Then, neglecting squares of small quantities, (2.1) becomes

$$
\phi_{\mathbf{l}_{\mathrm{v}}}=U \boldsymbol{\eta}_{\mathrm{i}}^{\prime}
$$

and (2.6) becomes

$$
g \eta_{1}-\Delta U \phi_{1_{x}}-\bar{T}_{\eta_{1}^{\prime \prime}}=0 .
$$

where

$$
\bar{T}=\frac{T}{\rho_{w}} .
$$

Upon elimination of $\eta_{1}$ between (3.3) and (3.4), we have

$$
g \phi_{1 y}=\Delta U^{2} \phi_{1_{x x}}+\bar{T} \phi_{1_{x x y}}
$$

as our free-surface condition for $\phi_{1}$ on $y=\eta_{0}$. Again, if we put $\Delta=-1$ we recover the usual condition for gravity-capillary water waves (Wehausen and Laitone [13], p. 632).

Plane waves of the form

$$
\phi_{1}=\mathscr{R} A e^{i k x-\imath \sigma t-k y}
$$

can exist in $y>\eta_{0}$, if $k>0$, and $k$ satisfies the dispersion relation

$$
g=\Delta U^{2} k-\bar{T} k^{2},
$$

which possesses solutions only if $U>U_{\min }$, where

$$
U_{\min }^{4}=\frac{4 \bar{T} g}{\Delta^{2}} .
$$

If $\Delta=-1$, this gives the normal minimum wave speed for gravity-capillary waves, which for an air-water interface takes a value

$$
\left[U_{\min }\right]_{(\Delta=-1)} \simeq 0.23 \mathrm{~ms}^{-1} \text {. }
$$

Correspondingly, if $\Delta \neq-1$,

$$
U_{\min }=\Delta^{-\frac{1}{2}} \cdot\left[U_{\min }\right]_{(\Delta=-1)} \simeq \dot{6} .5 \mathrm{~ms}^{-1},
$$

for an air-water boundary. This speed is of course well known (e.g. Ursell [12]) as the minimum velocity for Kelvin-Helmholtz instability to occur.

It is possible to utilize the whole range of steady linearized water-wave solutions, e.g. Wehausen and Laitone [13], pp. 568-591, to study aerodynamically-induced waves with $U>U_{\mathrm{mm}}$. In fact, we assume henceforth 
in the present paper that $U \gg U_{\min }$, so that surface tension can be neglected. It is not our purpose here to catalogue such possibilities. However, we may mention as an example that the theory of planing surfaces (Wehausen and Laitone [13], p. 587) can be inverted to provide the solution for air flow over a low barrier, or reef. The solution appears to imply a singularity at the leading or windward edge of the barrier and a system of waves trailing smoothly off its trailing or lee edge. Clearly some further work is required to clarify the true nature of the leading-edge flow, which in the planing-surface problem represents spray. Another example would be inversion of the classical solutions for hydrofoils just below the free surface (e.g. Wehausen and Laitone [13], p. 583) to provide solutions for airfoils operating just above the free surface, with possible applications to high-speed hovercraft, or related vehicles.

\section{Hodograph transformation of the free-surface condition}

As in the case of hydrodynamic water waves, solution of practical problems with the full non-linear free surface condition (2.6), on a surface $y=\eta(x)$ which is unknown, presents formidable difficulties. In the absence of surface tension ( $T=0$ ) we can, however, use a hodograph-type approach to reduce the flow region to a known space, as follows. If $u, v$ are the $x$ and $y$ components of velocity, then

where

$$
u-i v=f^{\prime}(z)=U e^{-i \Omega}
$$

$$
\Omega=i \log \left(f^{\prime}(z) / U\right)=\theta+i \tau .
$$

Thus $\theta$ is the angle between streamlines and $x$-axis, and

$$
|q|=U e^{\tau} .
$$

Thus (2.6) with $T=0$ implies

$$
g y-\frac{1}{2} \Delta U^{2} e^{2 r}=0
$$

on $y=\eta$, where $\psi=0$. We now treat $\phi, \psi$ as independent variables, with $y=y(\phi, \psi), \tau=\tau(\phi, \psi)$ in (4.4). Differentiating with respect to $\phi$ implies

But

$$
y_{\phi}=\frac{\Delta U^{2}}{g} e^{2 \tau} \tau_{\phi} .
$$

$$
y_{\phi}=\mathscr{I}\left(\frac{d z}{d f}\right)=\frac{1}{U} e^{-\tau} \quad \sin \theta,
$$

from (4.1). Hence (4.5) implies

$$
-\theta_{\psi}=\tau_{\phi}=\frac{g}{\Delta U^{3}} e^{-3 \tau} \sin \theta .
$$


Again the corresponding water-wave condition (Wehausen and Laitone [13], p. 728) is obtained by setting $\Delta=-1$ in equation (4.6).

Equation (4.6) can be integrated to give

$$
e^{3 \tau(\phi .0)}=e^{3 r\left(\phi_{0}, 0\right)}+\frac{3 g}{\Delta U^{3}} \int_{\phi_{0}}^{\phi} \sin \theta(\varphi, 0) d \varphi,
$$

which enables $\tau(\phi, 0)$ to be calculated for all $\phi$ values of the free surface, given the corresponding values of $\theta(\phi, 0)$ and the value of $\tau$ at one point $\phi=\phi_{0}$. For example, if $\phi=\phi_{0}$ is a stagnation point, $e^{3 \tau\left(\phi_{0}, 0\right)}=0$; alternatively if $\phi=\phi_{0}$ (usually $\phi_{0}=\infty$ ) is a point where the velocity takes the free-stream value $U$, then $\tau\left(\phi_{0}, 0\right)=0$. Transformations such as (4.7) with $\Delta=-1$ have been used, e.g. by Nekrasov [6], as the basis for existence proofs for periodic water waves.

The analytic character of the hodograph function $\Omega(f)$ depends on the presence of fixed bodies or other disturbing influences in the flow region $\psi \geqq 0$. For example, a stagnation point corresponds to a logarithmic singularity in $\Omega(f)$. After taking appropriate care of any such singularities, we can always use Cauchy's theorem in the upper-half $f$-plane to express $\Omega(f)$ in terms of its values on the real axis $\psi=0$. This provides a second integral expression for $\tau(\phi, 0)$ in terms of $\theta(\phi, 0)$, and hence in combination with (4.7) provides an integral equation for the unknown $\theta(\phi, 0)$.

As an example of a simple problem of this type, consider air flow over a semi-infinite solid boundary $x<0$ and a semi-infinite free water surface $x>0$. The problem can be set up as an integral equation in a semi-inverse manner, if we "specify" the shape of the solid surface by prescribing

$$
\theta(\phi, 0)=\Theta(\phi)=\text { given, } \phi<0 .
$$

Now in this case $\Omega(f)$ is analytic for all $\psi>0$ and tends to zero as $\psi \rightarrow+\infty$, so that Cauchy's theorem indicates that

$$
\tau(\phi, 0)=-\frac{1}{\pi} \int_{-\infty}^{\infty} \frac{\theta(\varphi, 0)}{\varphi-\phi} d \varphi,
$$

the integral taking a Cauchy principal value. Using (4.7) with $\phi_{0}=+\infty$, $\tau\left(\phi_{0}, 0\right)=0$, we have for $\phi>0$,

$$
\begin{aligned}
1-\frac{3 g}{\Delta U^{3}} \int_{\phi}^{\infty} \sin \theta(\varphi, 0) d \varphi & =e^{3 \tau(\phi, 0)} \\
& =Q(\phi) \exp \left(-\frac{3}{\pi} \int_{0}^{\infty} \frac{\theta(\varphi, 0)}{\varphi-\phi} d \varphi\right)
\end{aligned}
$$

where

$$
Q(\phi)=\exp \left(-\frac{3}{\pi} \int_{-\infty}^{0} \frac{\Theta(\varphi)}{\varphi-\phi} d \varphi\right)
$$


is a known function. A characteristic example is the stepped boundary

$$
\Theta(\phi)=\left\{\begin{array}{cc}
0, & \phi<-K \\
-\pi / 2, & -K<\phi<0,
\end{array}\right.
$$

for some parameter $K>0$ (which ultimately fixes the height of the step) which gives

$$
Q(\phi)=\left(\frac{\phi}{K+\phi}\right)^{\frac{3}{2}}
$$

More complicated problems can be reduced to integral equations similar to (4.10), after preliminary conformal mapping or other transformations. In particular, problems involving jets of air blowing on free surfaces can be treated in this way, the jet boundary being a constant-velocity streamline on which $\tau=0$. Such a reduction to a non-linear integral equation was carried out by Olmstead and Raynor [8], who also obtained numerical solutions of an approximate version of the integral equation, by trapezoidal discretization and Newtonian iteration.

The task of solving non-linear singular integral equations such as (4.10) is difficult and challenging from both the numerical and analytic points of view. Analytically, we are concerned with existence and uniqueness questions. Cleary as $g \rightarrow \infty$ or $U \rightarrow 0$, the free surface becomes flat and $\theta(\phi, 0) \equiv 0, \phi>0$, is the limiting solution. For sufficiently large non-dimensional gravity $g K / \Delta U^{3}$, or small Froude number $U \sqrt{\Delta / g L}$, where $L$ is a typical length scale, we should expect a solution to exist, and could obtain it by iteration about the $\theta=0$ limit. However, there is a real suspicion that there is a finite value of the Froude number above which no solution exists. The numerical results of Olmstead and Raynor [8] appear to indicate this effect, as does the physical fact that flows of this nature do tend to break up into spray if the air flow is sufficiently violent. There is thus some considerable value in establishing the limits on the Froude number for existence of a solution to integral equations such as (4.10). Such analysis would parallel existence work on periodic water waves, the limiting Froude number being analogous to the height/wavelength ratio of the highest wave. In the hydrodynamic case $(\Delta=-1)$, some such analysis of $(4.10)$ has been carried out by Oertel [7], as part of an investigation into bow waves of ships.

An inverse approach to (4.10) is perhaps of interest. We may ask whether we can find a solid-boundary function $\Theta(f), \phi<0$, which generates a "given" free surface $\theta(\phi, 0), \phi), \phi>0$. Thus $(4.10)$ is used with given $\theta(\phi, 0)$ as an equation to determine $Q(\phi)$, and (4.11) then becomes a Stieltjes integral equation

$$
\frac{1}{\pi} \int_{-\infty}^{0} \frac{\Theta(\varphi)}{\varphi-\phi} d \varphi=-\frac{1}{3} \log Q(\phi), \quad \phi>0,
$$


for the now-unknown $\Theta(\phi), \phi<0$. Although the integral equation. (4.14) is linear and non-singular, in pleasant contrast to (4.10), inversion of such Stieltjes transforms (Erdelyi [3]) is not at all straightforward, and it is not even certain if a solution exists for arbitrary $\theta(\phi, 0), \phi>0$.

\section{Exact inverse solutions}

Another use of the free surface condition (4.6) arises if we write from (4.1)

$$
\frac{d z}{d f}=\frac{1}{U} e^{-\tau}(\cos \theta+i \sin \theta)
$$

and use (4.6) to eliminate $\theta$, obtaining

$$
\frac{d z}{d f}=\frac{1}{U} e^{-\tau_{0}}\left[\sqrt{1-\left(\frac{\Delta U^{3}}{g} e^{3 \tau_{0}} \tau_{0}^{\prime}\right)^{2}}+i\left(\frac{\Delta U^{3}}{g} e^{3 \tau_{0}} \tau_{0}^{\prime}\right)\right],
$$

where $\tau_{0}(\phi)=\tau(\phi, 0)$. If now we allow $\tau_{0}(f)$ to be any analytic function, integration of (5.2) provides an analytic function $z(f)$ such that the free-surface condition is satisfied whenever both $\tau_{0}$ and the square root in (5.2) are real on $\psi=\mathbf{0}$.

Equation (5.2) thus provides an inverse procedure for generating freesurface flows. This method is entirely equivalent to the inverse methods of Sautreaux (e.g. [9]) and others (see Wehausen and Laitone, [13], p. 736) for the water-wave case $\Delta=-1$. The substitution

$$
Y(f)=\frac{\Delta U^{2}}{2 g} e^{2 \tau_{0}(f)}
$$

simplifies (5.2) to

$$
\frac{d z}{d f}=i Y^{\prime}(f)+\sqrt{\frac{\Delta}{2 g Y(f)}-\left(Y^{\prime}(f)\right)^{2}}
$$

which, with $\Delta=-1$, is a form given in Wehausen and Laitone ([13], p. 737). Equation (5.4) implies that $y=Y(\phi)$ on the free surface, and can therefore be obtained rather more directly using (4.4).

In principle, an unlimited range of inverse solutions are available to us by suitable choice of $Y(f)$ in (5.4). Unfortunately, almost any choice beyond the simplest analytic functions introduces non-physical branch-point singularities into the region of flow. For example, if the quantity under the square root in (5.4) possesses a simple zero at $f=f_{0}$, corresponding to $z=z_{0}$ in the physical plane, then

$$
f-f_{0}=a\left(z-z_{0}\right)+b\left(z-z_{0}\right)^{\frac{3}{2}}+0\left(z-z_{0}\right)^{2}
$$


for some constants $a, b$. Streamlines through $z=z_{0}$ bifurcate, and the complex plane is doubly covered near $z=z_{0}$. In some but not all such circumstances, one can simply remove this point by choosing as an upper boundary to the flow, a streamline which passes below the offending point. On the other hand, simple poles in the quantity $\Delta / 2 g Y-Y^{\prime 2}$ correspond to stagnation points, and are quite acceptable.

Most useful simple choices for $Y(f)$ have been summarized by Wehausen and Laitone ([13], pp. 738-740) for the hydrodynamic case $\Delta=-1$. As we should expect from the correspondence principle (2.13), every such hydrodynamic solution has its aerodynamic $(\Delta>0)$ counterpart, simply obtained by reversing signs of $x, y$, and $\eta$, and scaling velocities by the factor $\Delta^{-\frac{1}{2}}$.

The effect of this correspondence principle on the Sautreaux formula (5.4) is that if we have already computed for $\psi=\mathscr{I}_{f} \leqq 0$ a set of hydrodynamic streamlines $z=z_{-}(f)$, where

$$
\frac{d z_{-}}{d f}=i Y_{-}^{\prime}(f)+\sqrt{-\frac{1}{2 g Y_{-}(f)}-\left(Y_{-}^{\prime}(f)\right)^{2}},
$$

for some given function $Y_{-}(f)$ which is analytic in $\psi \leqq 0$, then

$$
z=-z_{-}\left(-\Delta^{\frac{1}{2}} f\right)
$$

defines streamlines in $\psi \geqq 0$, and satisfies (5.4) with

$$
Y(f)=-Y_{-}\left(-\Delta^{\frac{1}{2}} f\right) .
$$

For example, the choice

$$
Y_{-}(f)=a f
$$

for some positive real constant $a$, generates a hydrodynamic "fountain", which on turning upside down according to (5.7) becomes a rising and falling stream of air above a stationary water surface, between two vertical walls. Similarly, the choice

$$
Y_{-}(f)=a f^{\frac{1}{2}}
$$

generates for $\Delta=-1$ a flow of water under a vertical "sluice gate", and for $\Delta>0$ a flow of air over a vertical semi-infinite barrier holding back stationary water.

The choice

$$
Y(f)=a e^{b f}
$$

in (5.4) leads for $\Delta>0$ to the result

$$
z(f)=a \gamma Z(b f-\log \gamma)
$$


where

and

$$
\gamma=\left(\frac{\Delta}{2 g a^{3} b}\right)^{\frac{1}{2}}
$$

$$
Z^{\prime}(F)=i e^{F}+e^{F} \sqrt{-1+e^{-3 F}} .
$$

A set of streamlines $\Psi=\mathscr{I F} \geqq 0$, computed by integration of (5.14), is shown in Fig. 1. Although a choice of $Y_{-}(f)$ equivalent to (5.11) was made by Sautreaux [9], hydrodynamic streamlines equivalent to an inversion of Fig. 1 do not seem to have previously published.

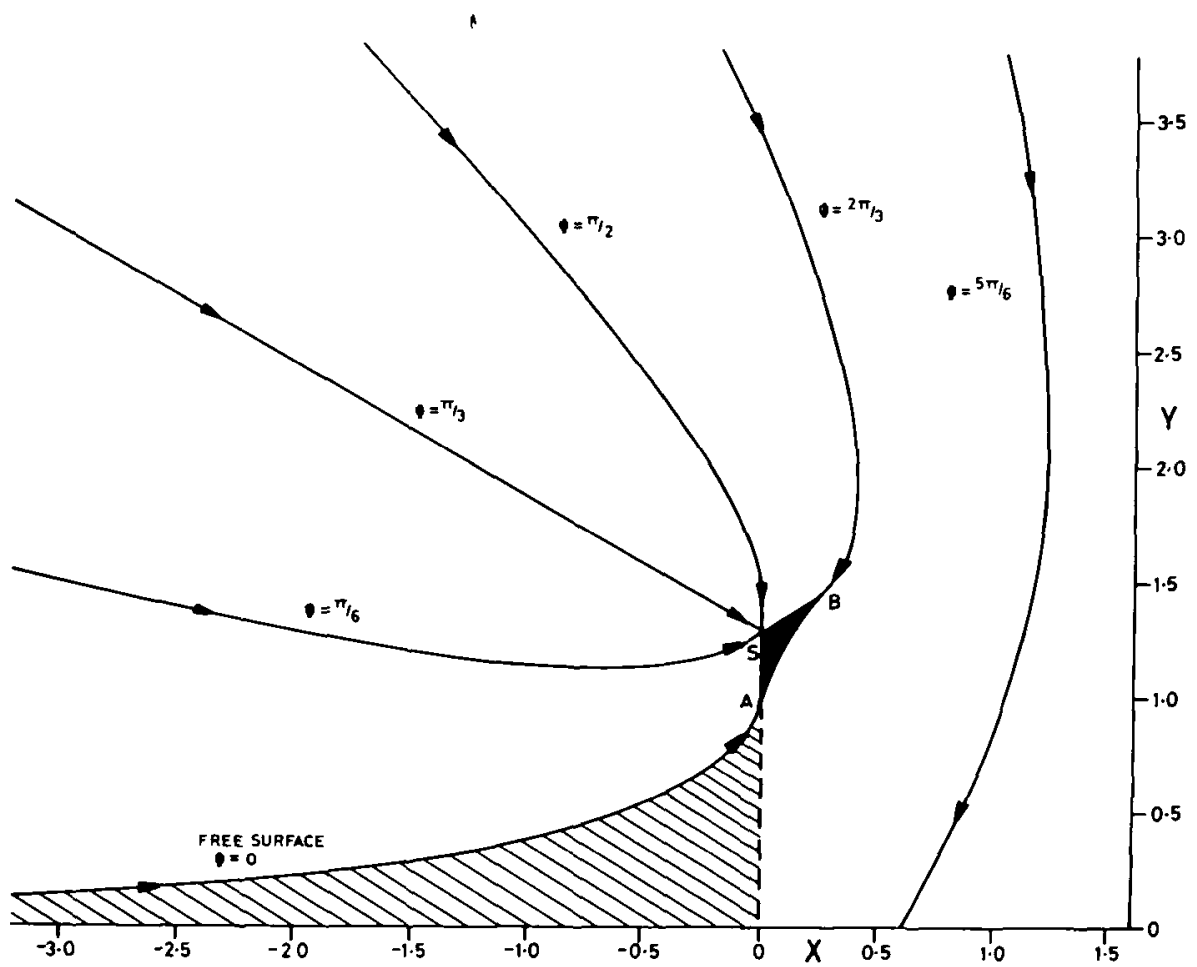

Fig. 1.- Streamlines of air flow into a sink at $S$. The streamline $\Psi=0$ consists of a free water surface from $-\infty$ to $A$, and a vertical wall from $A$ to $S$. The streamline $\Psi=2 \pi / 3$ bifurcates at $B$, one branch entering $S$ and the other passing through $A$ onto a second flow sheet, not shown.

The flow of Fig. 1 consists of a radial streaming from infinity into a sink at $Z \simeq 1.29 i$. The free surface extends from $Z=-\infty+i 0$ to $Z=i$, the remainder of the streamline $\Psi=0$ being a vertical wall from $Z=i$ to $Z=1.29 i$. 
Streamlines are single-valued only for $\Psi \leqq 2 \pi / 3$, there being a bifurcation point $B$ at $z(2 \pi i / 3) \simeq 0.25+1.43 i$. The streamline $\Psi=\pi / 3$ is a straight line and the flow $0 \leqq \Psi \leqq 2 \pi / 3$ is symmetrical about this line. Either of the streamlines $\Psi=\pi / 3$ and $\Psi=\pi / 2$ are good choices for upper solid boundaries, the former because it is straight, the latter because it meets the vertical wall smoothly. The sink itself can mode! a smal! slit through which the air escanes (c.f. Tuck [10]).

One further simple case, which has apparently not been studied even for $\Delta=-1$, is

$$
Y(f)=a-b f^{2},
$$

for which (5.4) gives

$$
z^{\prime}=-2 i b f+\left(a-b f^{2}\right)^{-\frac{1}{2}}\left[\frac{\Delta}{2 g}-4 a b^{2} f^{2}+4 b^{3} f^{4}\right]^{\frac{1}{2}} .
$$

Although for general $a, b$ each distinct zero of the square bracket in (5.16) represents an unacceptable bifurcation point, in the special case where

$$
a^{2} b=\frac{\Delta}{2 g}
$$

the zeros coalesce and we have

$$
z^{\prime}=-2 i b f+b^{\frac{1}{2}}\left(a-2 b f^{2}\right)\left(a-b f^{2}\right)^{-\frac{1}{2}},
$$

which integrates to give

$$
\begin{aligned}
z & =i\left(a-b f^{2}\right)+b^{\frac{1}{2}} f\left(a-b f^{2}\right)^{\frac{1}{2}} \\
& =a Z\left(\sqrt{\frac{b}{a}} f\right),
\end{aligned}
$$

where

$$
Z(F)=i\left(1-F^{2}\right)+F\left(1-F^{2}\right)^{\frac{1}{2}}
$$

or

$$
F(Z)=(1+i Z)(1+2 i Z)^{-\frac{1}{2}} .
$$

Streamlines $\Psi=\mathscr{I} F \geqq 0$ corresponding to (5.20) are shown in Figure 2, the free surface $\Psi=0$ being the circle $\left|Z-\frac{1}{2} i\right|=\frac{1}{2}$. Streamlines at infinity are asymptotically parabolic with a vertical axis. Thus the flow represents air streaming around a vertical semi-infinite plate, near whose top edge is a cylindrical bubble of stationary water. The bubble is held up by the stagnation pressure at its contact points with the plate, the aerodynamic pressure around its circular section then varying linearly with height, so as to provide precisely what is required to balance hydrostatic pressure in the water. 


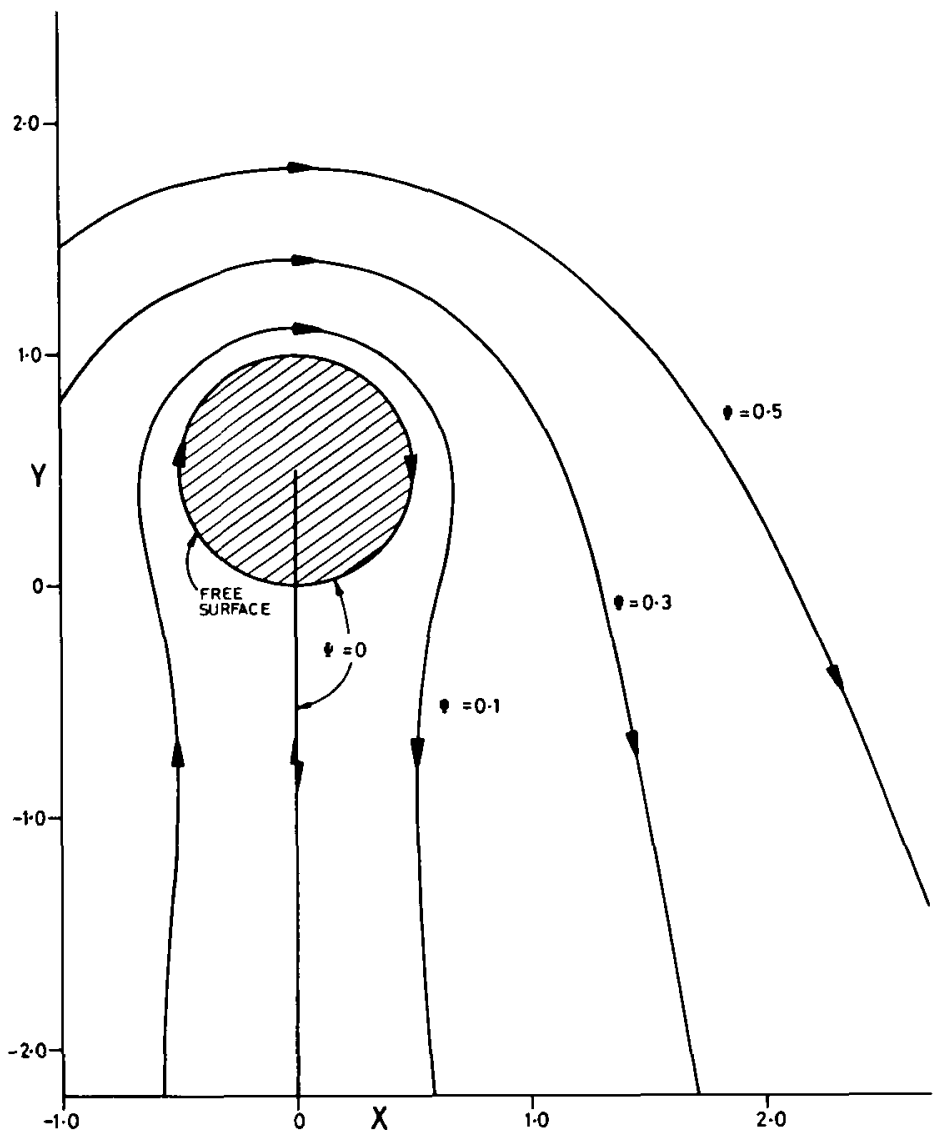

Fig. 2.- Streamlines for air flow around a semı-infinite vertical plate, with a cylindrical bubble of stationary water at its edge.

The above exact solutions are to a large extent mathematical curiosities, the boundary geometries being remote from practical applications, and the flows themselves possessing features which are unlikely to be realizeable for real fluids with viscosity, surface tension, etc. However, these results at least demonstrate the existence of solutions to potential-flow problems involving non-linear free surfaces under gravity. In order to attack more practical problems, one must attempt solutions of integral equations such as (4.10), and work is proceeding on this task. 


\section{References}

[1] J. Berghmans, 'Theoretical investigation of the interfacial stability of inviscid fluids in motion, considering surface tension', J. Fluid Mech. 54 (1972), 129-141.

[2] L. J. Doctors, 'The forces on an air cushion vehicle executing an unsteady motion', 9th Symp. Naval Hydro., Paris. Proceedings, O. N. R., Wash. D. C. (1972).

[3] A. Erdelyi (Ed.) Tables of integral transforms, Vol. II, McGraw-Hill (1954).

[4] B. V. Hamon, 'Continental shelf waves and the effects of atmospheric pressure and wind stress on sea level', J. Geophys. Res. 71 (1966), 2883-2893.

[5] T. K. S. Murthy, The static depression of a hovercraft cushion and of the peripherical jets over water, University of Southampton, A. A. S. U. Report No. 297 (1970).

[6] A. I. Nekrasov, 'On waves of permanent type I', Izv. Ivanovo-Voznesensk Politekhn. Inst. 3 (1921), 52-65.

[7] R. Oertel, The steady motion of a flat ship, with an investigation of the flow near the bow and stern. Ph. D. thesis, University of Adelaide (1975).

[8] W. E. Olmstead and S. Raynor, 'Depression of an infinite liquid surface by an incompressible gas jet', J. Fluid Mech. 19, (1964), 561-576.

[9] C. Sautreaux, 'Mouvement d'un liquide parfait soumis à la presanteur. Determination des lignes de courant', J. Math. pures appl. (5) 7 (1901), 125-159.

[10] E. O. Tuck, 'Matching problems involving flows through small holes', in Advances in Applied Mechanics (ed. C. S. Yih), Vol. 15, 89-158, Academic Press (1975).

[11] E. T. Turkdogan, 'Fluid dynamics of gas jets impinging on surface of liquids', Chem. Eng. Sci. 21 (1966), 1133-1144.

[12] F. Ursell, 'Wave generation by wind', in Surveys in Mechanics (eds. G. K. Batchelor and R. M. Davies), Cambridge University Press (1956).

[13] J. V. Wehausen and E. V. Laitone, 'Surface waves', in Handbuch der Physik (ed. S. Flugge) Vol. 9, Springer.

\section{Department of Applied Mathematics}

University of Adelaide

Adelaide, S. A. 5000

Australia. 